\title{
The European Extremely Large Telescope : science case and project status
}

\section{Hook*}

U. Oxford Astrophysics, UK and INAF-Obs. Rome, Italy

E-mail: imheastro.ox.ac.uk

The 42m European Extremely Large Telescope (E-ELT) project is currently nearing the end of the detailed design stage, and is due to begin operations towards the end of this decade. In this paper an overview of the science case is presented, concentrating on cases that include a component of time-resolved observations. Finally a summary of the status of the project is presented.

High Time Resolution Astrophysics IV - The Era of Extremely Large Telescopes - HTRA-IV,

May 5-7, 2010

Agios Nikolaos, Crete, Greece

${ }^{*}$ Speaker. 


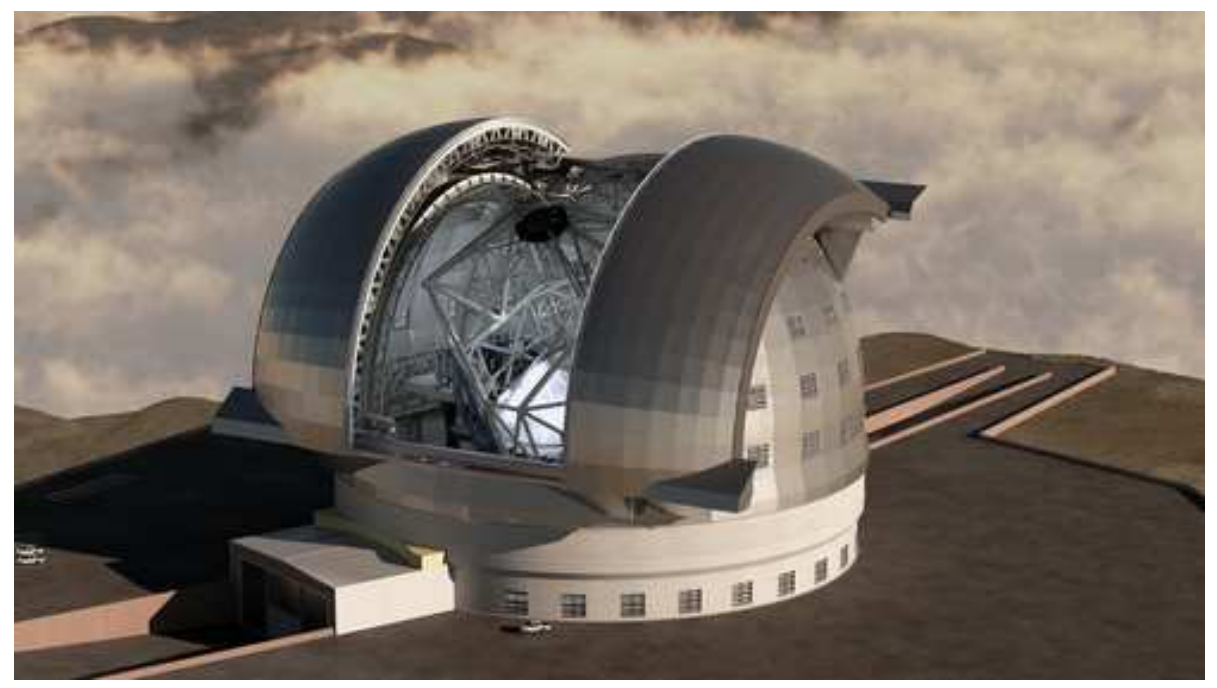

Figure 1: A rendering of the European Extremely Large Telescope. Credit: Swinburne Astronomy Productions/ESO

\section{Introduction}

The European Extremely Large Telescope (E-ELT) project aims to design and construct a $42 \mathrm{~m}$ diameter, adaptive telescope, which will be the largest optical/IR telescope in the World. Such a telescope will enable spectacular new science and complements other flagship facilities that are expected to be operating on a similar timescale, including VLT(I), JWST, ALMA, IXO and SKA. The project is currently nearing the end of the detailed design phase, with a plan to start construction in 2011, and first light 7-8 years later. The E-ELT has been identified as a top priority of European ground-based astronomy by the ASTRONET roadmapping exercise[1]. Figure 1 shows a rendering of the completed telescope and dome.

Two other major international ELT projects are also under development. The Thirty Meter Telescope (TMT) project (with partners U. California, Caltech, ACURA(Canada) in collaboration with NAOJ (Japan) and additionally China and recently India with observer status) has recently completed a construction proposal, and is aiming to start science operations in 2018. The Giant Magellan Telescope (GMT) project team, consisting of a collaboration of private US universities, Australia (the Australian National University and Astronomy Australia Ltd) and the Korea Astronomy and Space Science Insitute, are designing a 24m-diameter telescope. The first of GMT's seven $8 \mathrm{~m}$ mirror segments was sucessfully cast in 2005 and first light for the telescope is also projected for around 2018.

\subsection{E-ELT performance}

In simple terms, a large, filled-aperture telescope gains over smaller telscopes in two ways. Firstly the increased collecting area leads to enhanced sensitivity, and secondly the larger diameter results in higher resolution, assuming that adaptive optics (AO) is used to produce images close to the diffraction limit of the telescope. The combination of these two factors mean that in 
Table 1: E-ELT imaging point source $5 \sigma$ sensitivity in $1 \mathrm{hr}$, using the Laser Tomography AO mode (LTAO) or the Ground Layer AO mode (GLAO). The limiting magnitudes on the vega system are given to the nearest $0.5 \mathrm{mag}$. The values were calculated using the E-ELT ETC, with S/N reference area chosen to best match the simulated AO PSF. In some cases, marked with *, the 5mas pixel scale is not well matched to PSF. Values in brackets indicate that the adaptive optics systems are not expected to deliver diffraction-limited images at these wavlenegths. Table compiled by Chris Evans.

\begin{tabular}{cccc}
\hline Band & $\begin{array}{c}\lambda / D \\
(\mathrm{mas})\end{array}$ & $\begin{array}{c}\text { E-ELT with LTAO } \\
(5 \text { mas pixels })\end{array}$ & $\begin{array}{c}\text { E-ELT with GLAO } \\
\text { (50 mas pixels) }\end{array}$ \\
\hline $\mathrm{V}$ & $(2.7)$ & $(27.5)^{*}$ & 29.0 \\
$\mathrm{R}$ & $(3.1)$ & $(28.5)^{*}$ & 29.0 \\
$\mathrm{I}$ & 3.9 & 29.5 & 28.5 \\
$\mathrm{~J}$ & 6.1 & 28.5 & 26.0 \\
$\mathrm{H}$ & 8.1 & 28.0 & 25.0 \\
$\mathrm{~K}$ & 10.6 & 27.5 & 24.5 \\
\hline
\end{tabular}

some regimes, particularly background-limited observations of point sources, the gains are truly dramatic, with exposure time scaling as $\mathrm{D}^{-4}$, where $\mathrm{D}$ is the telescope diameter.

Table 1 gives the expected point source sensitivity for the E-ELT operating with either of two AO modes. Some simple scaling arguments can be used to estimate the sensitivity during short exposures. For example, if the observations are background limited and hence readnoise can be ignored, the $5 \sigma$ sensitivity in 1 second can be obtained by subtracting 4.4 mag from the limiting magnitides in Table 1.

The E-ELT collecting area is roughly 25 times that of an $8 \mathrm{~m}$ telescope. Since exposure time sclates as $\mathrm{D}^{-2}$ in many regimes (including seeing-limited observations with no $\mathrm{AO}$ ), this implies that with E-ELT we can observe the same sources with the same $\mathrm{S} / \mathrm{N}$ a factor of 25 times faster than is possible with an $8 \mathrm{~m}$ telescope. Alternatively one could do spectroscopy or polarimetry where an $8 \mathrm{~m}$ can do imaging.

As mentioned above, even bigger gains can be made for AO-corrected observations of point sources in the background-limited regime. Here $\mathrm{T}_{\exp } \propto \mathrm{D}^{-4}$, implying a gain of a factor of 750 in exposure time. This implies that for the same $\mathrm{S} / \mathrm{N}$, exposures could be taken a factor of 750 times faster. However it should be noted that these simple scaling arguments ignore the effects of readnoise and dead time between exposures.

\section{Time resolved science cases}

The science case for the E-ELT has been developed over several years by a large section of European community. Meetings arranged by OPTICON and ESO have been central to coordinating this effort. The connection to the project has been maintained by the Science Working Group (SWG) and the Project Scientist at ESO.

A summary of the science case is publically available in a report of the SWG from 2006[2]. Of the science cases included in that document, many have a time-resolved component. Some examples are listed below. 
- Solar system: monitoring weather and volcanic activity

- Exo-planets: radial velocity, direct imaging and transit measurements

- The Galactic Centre: orbits of stars close to the central black hole

- Supernovae: lightcurves and specra of transient events

- Gamma Ray Bursts

- Expansion of the Universe: measuring the effects of Dark Energy in real time

A few of these cases are expanded below.

\subsection{Exoplanets}

\subsubsection{Direct Detection}

The exquisite spatial resolution of the E-ELT will make it a powerful tool for direct detection and characterisation of exoplanets, with the potential to push the observable range towards older giant planets seen in reflected light and ultimately even a small number of rocky planets in habitable zones. A specialised instrument concept is being developed (EPICS,[3]) that is optimised for the detection and characterization of expolanets by direct imaging and spectroscopy. It will employ extereme $\mathrm{AO}$ and a combination of techniques for suppression of speckles in the Near-IR (including polarimetry and spectral suppression techniques using an IFS) to acheive the highest possible contrast ratio, approaching $10^{9}$ contrast between the planet and its parent star.

This technique will be complemented by detection of exoplanets at mid-IR wavelengths using a suitable AO-fed instrument (such as METIS, which was studied for the E-ELT, [4]). Although the PSF is broader at longer wavelengths, the intrinsic contrast between the parent star and planet is lower, hence the observations at near- and mid-IR wavelengths are largely complementary. At mid-IR wavelengths the E-ELT will be able to detect self-luminous planets of a few Jupiter masses (see [5] for example simulations).

In cases where a new planet is discovered, observations at multiple epochs are required for confirmation that the planet is associated with the parent star, and for measurement of its orbit.

\subsubsection{Atmospheres}

In addition to the information provided by direct detection, transiting planets provide the opportunity to measure fetaures in their atmospheres by subtracting spectra when the planet is in and out of transit. In the case of planets in the habitable zone, it may even be possible to detect signs of life in the spectrum of the atmosphere. This is one of the key science case for a NIR echelle spectrograph on the ELT (such as the SIMPLE concept [6]).

Such measurements require multiple observations of the transiting system, so that spectra from multiple transits can be combined. In the case of a planet in a habitable orbit around an $\mathrm{M}$ dwarf star, the eclipse duration would be of order $0.5 \mathrm{hr}$, and the orbital period 2-6 days. Observing an Earth-like planet in this way would be significantly more challenging, requiring combination of data from $\sim 100$ transits, with observations required 1-2 times per week for 2 years. 


\subsubsection{Indirect detection: Radial Velocity}

The radial velocity method for detecting exo-planets has provided the majority of known exoplanets to date. The E-ELT, equipped with a suitable ultra-stable spectrograph such as CODEX [7], haas the potential to push this technique to reach rocky planets in habitable zones. This requires reaching the order of $\mathrm{cm} / \mathrm{s}$ precision accuracy in radial velocity measurements, stable over periods of many years, which in turn requires both the improved S/N offered by the E-ELT's collecting area and also developments in calibration techniques (for example the laser comb for wavelength calibration which is currently being developed and tested).

Observations at multiple timescales are required to overcome systematic effects (such as stellar activity). A typical observing scenario might involve 3-4 observations per night, repeated every night for 10 nights. This 10-night pattern would then be repeated every month that the target is visible over a monitoring timescale of about 2 years, see e.g. [8].

In addition to radial velocity measurements in the optical, NIR observations have the advantage of being sensitive to lower mass stars which are intrinsically very red, and hence too faint in the optical. For the same mass of planet, the radial velocity signal is larger for low mass stars, making it easier to detect Earth-mass planets in habitable zones. A high resolution NIR spectrograph on the E-ELT would be capable of searching for such systems to a distance of 200pc [6].

\subsection{Galactic Centre(s)}

Detailed study of the centre of our own Galaxy and the centres of other nearby galaxies is a key science case for a high-resolution imager (such as the MICADO instrument that has been studied for the E-ELT[9]). In our own Galactic centre, precise astrometric measurements of stars orbiting $\mathrm{SgA}^{*}$ have produced evidence that the Galactic centre contains a massive black hole, with mass of order $4 \times 10^{6}$ solar masses[10]. This region provides an ideal location in which to study the effects of strong gravity and accretion on to black holes.

Currently abdout 30 bright stars observable near SgrA* (using AO systems on Keck and VLT). The E-ELT is likely to detect stars more than five magnitudes fainter, and closer to the black hole, with velocities approaching 0.1c. In order to detect the effects of Special and General Relativity requires long-term astrometric precision of order $50 \mu$ as and proper motion accuracy of order $10 \mu \mathrm{as} / \mathrm{yr}$ (see [11] for details).

\subsection{Cosmology}

\subsubsection{Type Ia Supernovae}

Current measurements of Dark Energy equation of state parameter are consistent with $\mathrm{w}=-1$ to about 5\% statistical uncertainty[12]. The behaviour of Dark Energy appears to be consistent with a cosmological constant $(\Lambda)$, but the origin of such a vacuum energy is not understood. Several new experiments are now running or being planned in order to improve our constraints on the nature of Dark Energy, including new surveys that will discover hundreds of nearby supernovae (such as PanStarrs, Skymapper and Palomar Transient Factry) and more distant supernovae with redshifts up to $z<1.7$ (via proposed space missions such as JDEM and EUCLID). However in order to reach even higher redshifts, the next generation of space and ground-based telescopes is required. 

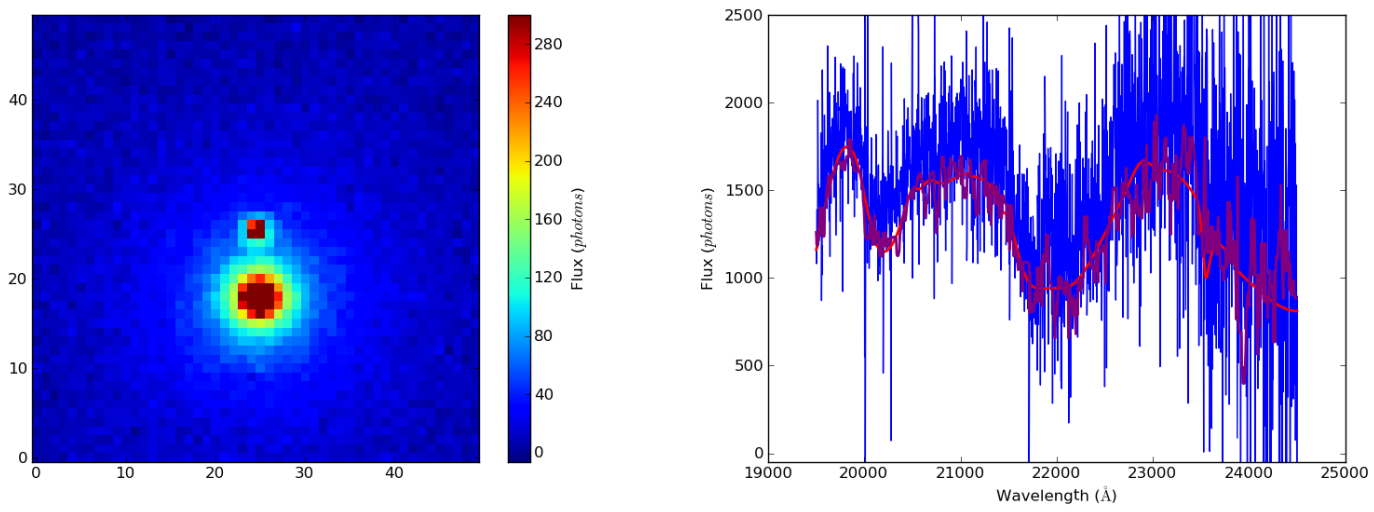

Figure 2: Left: Simulated E-ELT 8-hour K-band observation with the HARMONI integral field spectrograph of a distant SN (point source, centre) placed on simulated host galaxy (below). The image represents the data cube after collapsing along the wavelength direction by taking a median. Right: the blue line shows the integrated spectrum from the four brightest pixels in the supernova. The red line shows the noise-less photon counts for the supernova without host contamination (i.e. an input to the simulation). The purple line shows a median-filtered spectrum of the supernova light with a very coarse removal of the host contamination. As can be seen the extracted, host-free spectrum follows the true supernova light closely, and the SiII broad feature (centred at observed wavelength $\lambda \sim 2.0 \mu \mathrm{m}$ ) is clearly detected, implying that E-ELT can confirm the SN type at $z=4$. Image credit: Tim Goodsall and the HARMONI team.

Simulations show that the E-ELT, equipped with a suitable spectrograph (such as HARMONI, [13]), would be capable of obtaining spectra of Type Ia supernovae (SNeIa) and confirming their type and redshift, out to $z=4$ (see Fig 2). These SNeIa could be discovered and photometrically monitored in an imaging survey with JWST, and when these data are combined with E-ELT redshift and Type determinations, the SNeIa can be used as distance indicators for cosmology. Spectroscopic follow-up of the SNeIa should take place when the SNe are close to peak brightness, hence these are time-critical observations within a window of a one or two weeks (depending on redshift).

\subsubsection{The expansion of the Universe}

A novel way to test the nature of Dark Energy will become possible with the E-ELT, which is to measure the acceleration of the Universe directly, in real time. The technique involves very accurate measurement of the redshifts of Ly $\alpha$ absorption lines in the spectra of background QSOs. By repeating these measurements over a long period (10-20yrs) the shift in redshift due to the acceleration of the Universe can be measured[14]. This is a fundamentally different probe of Dark Energy in that it is a dynamical measurement, whereas other measurements such as those from SNeIa and weak lensing are geometrical. However the signal to be detected is very weak, of order a few $\mathrm{cm} / \mathrm{s} / \mathrm{yr}$. This experiment requires the collecting area of the E-ELT to provide sufficient signal to noise, and an Ultra-high stability, high-resolution spectrograph (such as the CODEX concept that has been studied for the E-ELT, [7]). 


\subsection{HTRA science case}

The obove cases are examples of relatively "low time resolution astrophysics"that could be carried out with the E-ELT. However the E-ELT clearly also opens up parameter space for HTRA by providing very high sensitivity. The case for HTRA on the ELT has been summarised in a document [15] recently delivered to the project. It shows how, with sub-minute and sub-second resolution, one can study, for example, extreme physics (e.g. pulsars, neutron stars, black holes) and stellar phenomena (GRBs, transits and occultations).

In addition to high time resolution observations on ELT with a suitable instrument, ELT will also further the field of HTRA more generally by providing identification of new sources discovered at other wavelengths, including transients and other rapidly varying sources.

\section{Project status and timeline}

\subsubsection{Telescope design and site}

The E-ELT design consists of a Nasmyth telescope with a $42 \mathrm{~m}$ diameter primary mirror. Instruments and post-focal AO systems will be mounted on two instrument platforms the size of tennis courts, at the sides of the primary mirror. The design is a novel 5 mirror design to include adaptive optics in the telescope, and will make use of laser guide stars. The design provides outstanding image quality.

Several sites have been tested, including sites in the Canary Islands, Chile, Morocco, Argentina and Mexico. Over the last couple of years a down-selection was made to four sites : La Palma and three sites in Chile. The selection criteria include impact on science, outstanding atmosphere, but also construction and operations logistics (roads, water, electricity, nearby cities etc). On 26 April 2010 ESO Council approved the selection of Cerro Armazones in Chile as the baseline site for the E-ELT. This site is at an altitude of 3060 metres in the Atacama Desert and about 20 kilometres from the VLT site at Cerro Paranal.

\subsubsection{Instrumentation}

Phase-A studies for E-ELT instrumentation have been carried out in collaboration with institutes in ESO community. Eight instruments and two AO modules were studied, and the last of the studies was completed in Spring 2010. Two or three of these are expected to be selected for first light, and the full first generation instrument suite of about 6 instruments will be built up over first decade. The selection process for the instruments is underway now. A list of instrument Phase A studies is given in Table 2.

Possibilities exist for implementing HTRA detectors within some of these instrument concepts. When discussing instrumentation priorities, the SWG has encouraged these possibilities to be explored further. The SWG also supports the the possibility of a visitor focus in the future (after 1st generation) for specialised instruments.

\subsubsection{Conclusions and next steps}

The Phase B study of the telescope will be complete by the end of 2010, and a construction proposal will be ready to be presented to ESO Council in December 2010. This will include cost 
Table 2: List of E-ELT instrument Phase A studies

\begin{tabular}{ll}
\hline Instrument name & Description \\
\hline EAGLE & Multi-IFU, AO-fed near-IR spectrometer \\
EPICS & Extreme-AO imager/spectro-polarimeter for exo-planets \\
HARMONI & Diffraction-limited, near-IR IFU with optical extension \\
METIS & Mid-IR $(3-14 \mu \mathrm{m})$ imager and spectrometer \\
OPTIMOS & Seeing-limited/GLAO high-multiplex spectrograph \\
CODEX & Ultra-high-resolution optical spectrograph \\
MICADO & Near-IR, high-resolution imaging camera \\
SIMPLE & Near-IR, high-resolution spectrograph \\
\hline MAORY & Multi-conjugate AO relay \\
ATLAS & Laser Tomography AO relay \\
\hline
\end{tabular}

estimates based on industrial studies. The proposal will also include an instrumentation plan, which is now under development. If approved in December, the E-ELT could see first light in about 2018. For more information please see the following sites.

- The science web pages: http://www.eso.org/sci/facilities/eelt/

- The public web pages http://www.eso.org/public/astronomy/projects/e-elt.html

- Brochures, Posters, etc: http://www.eso.org/public/outreach/products/publ/brochures/ir

- Gallery: http://www.eso.org/gallery/v/ESOPIA/EELT

\section{References}

[1] The ASTRONET Infrastructure Roadmap, www.astronet-eu.org/IMG/pdf/Astronet-Book.pdf

[2] Report of the E-ELT Science Working Group, M. Franx (ed), 2006, available at http://www.eso.org/sci/facilities/eelt/publications.html

[3] Verinaud C et al, in "Ground-based and Airborne Instrumentation for Astronomy II". Edited by McLean, Ian S.; Casali, Mark M. Proceedings of the SPIE, Volume 7014, pp. 70141J-70141J-12 (2008)

[4] Kendrew S et al, Proc SPIE "Ground-based and Airborne Instrumentation for Astronomy III (2010)", Vol 7735

[5] Hippler S et al, AIP conf Proc "Exoplanets and disks: their formation and diversity", Vol 1158, p 333 (2009)

[6] Origlia L and Oliva E, Proc conference "Earth, Moon and Planets", Publisher Springer Netherlands Volume 105, Numbers 2-4 / September, 2009, p123-126

[7] Pasquini L et al in "Ground-based and Airborne Instrumentation for Astronomy II". Edited by McLean, Ian S.; Casali, Mark M. Proceedings of the SPIE, Volume 7014, pp. 70141I-70141I-9 (2008).

[8] Naef D, Dumusque X, Udry S, Lovis C, "The E-ELT Design Reference Mission: Earth Twins in the Habitable Zone of Solar-Type Stars Results of Simulations", available at http://www.eso.org/sci/facilities/eelt/science/drm/S3/ 
[9] Davies R and the MICADO team, Proc SPIE 7735-80 (Jun 2010)

[10] Gillessen et al 2009, ApJ, 692, 1075

[11] Trippe S, et al, MNRAS, 402, 1126

[12] Sullivan et al, the SNLS collaboration, in prep

[13] Tecza M, Thatte N, Clarke F, Freeman D., Proc "Science with the VLT in the ELT Era", Astrophysics and Space Science Proceedings, Springer Netherlands, 2009, p. 267

[14] Liske J et al 2008, MNRAS, 386, 1192

[15] "A science case for high time resolution astrophysics with the E-ELT", 2010, OPTICON HTRA network, this volume. 